\title{
LAS GRIETAS EN LAS ESTRUCTURAS DE FÁBRICA. UN PROCEDIMIENTO PARA EVALUAR LA FORMA DE TRABAJO DE ÉSTAS
}

\author{
(CRACKS IN MASONRY. A PROCEDURES FOR EVALUATION)
}

Antonio-José Mas-Guindal Lafarga, Dr. Arquitecto

Prof. Titular de la ETSAM. Asesor de la Fundación Cultural COAM

ESPAÑA

Fecha de recepción: 25-X-96 $631-15$

\section{RESUMEN}

Tradicionalmente, las estructuras de fábrica han sido evaluadas a partir de su geometría original, sin contar, a veces, con levantamientos precisos, partiendo de modelos ideales. Recientemente, se ha comprobado que la naturaleza de estas estructuras no permite un análisis elástico, dada su constitución hiperestática alta, y que el conocimiento de la geometría deformada de estas estructuras es crucial para poder establecer conjeturas acerca de su funcionamiento, bien se trate su solución a través de métodos basados en la teoría plástica, elástica (métodos numéricos), combinación de ambas o, simplemente, por aplicación de métodos gráficos.

El presente articulo, se propone, de forma resumida, evidenciar estas diferencias en la forma de enfocar el análisis de los edificios de fábrica, a partir de la geometría trasladada por la fisuración, fenómeno de aparición obligada en toda estructura de fábrica que está trabajando.

\section{SUMMARY}

Tradicionally the masonry structures, are evaluate to start from its original geometry, without to rely on, sometimes, with precious lifthings, starting to the ideals models. Recently, on the checking, that the nature of these structures not mermit a elastic analysis, since as it constitution hyperestatique is high, and that the knowledge og the deformed geometry of these structures is basic, to can stablish conjectures about his working, well though of methods to based in plastics theories, elastics (numeriques methods), combinations of both o simply for aplications of grafhics methods.

The present paper, on propose in the summarize form show, theses diffrerences in the form to approach the analysis of masonry buildings, to star of removed geometry for the cracks, phenomenon of the obligate apparition in all masonry structure that they are working.

\section{INTRODUCCIÓN}

Las fisuras o grietas (fisuras grandes) pueden definirse como la manifestación de la rotura del material, por incapacidad de asumir tracciones, dicho de otro modo, las fisuras sólo indican la aparición -no deseada- de tracciones en puntos de la estructura no preparados para asumirlas. Estas tracciones pueden proceder, bien de un estado de 
tracción o de un estado de compresión, en este caso, las tracciones se generan en sentido normal a la compresión.(Fig. 1)

Toda estructura, para trabajar, debe acercar unas moléculas a otras (deformación por compresión), o separar otras entre sí (deformación por tracción); este hecho, en materiales tenaces como el acero es difícil de observar, ya que en los estados de servicio, el material aporta ductilidad suficiente para que la fisuración no aparezca, pero, en materiales no cohesivos, aun en estados de servicio, lo habitual es manifestar su forma de trabajar a través de fisuras y grietas. Así pues, de la forma de las fisuras, pueden obtenerse las causas que las produjeron y actuar en consecuencia. Todas las arquitecturas y obras civiles de fábrica manifiestan su daño a través de fisuras, de formas y evaluaciones diversas.

Más importante que la propia grieta es su evolución. Si se parte del hecho de que todas las construcciones, en mayor o menor medida están fisuradas, las fábricas, de forma inequívoca, por la naturaleza de su constitución (material pétreo más aglomerante, mortero, cal, yeso), lo están, de ahí que sea especialmente importante dictaminar correctamente sobre la causa y posible evolución de las fisuras en toda estructura a reparar. Este fenómeno común no debe de llevar a diagnósticos de ruina en el edificio que se refuerza. En principio, una estructura, total o parcialmente fisurada, sólo indica que está trabajando de forma distinta a como se pensó en origen, sin querer, con esta afirmación, asegurar que una estructura fisurada no pueda estar al borde del colapso.

Las fisuras han sido motivo de multitud de diagnósticos de estructuras que, muchas veces, de forma equivocada, han llevado a la demolición del elemento o de la totalidad, por desconocimiento de su causa mecánica y, en consecuencia, por la imposibilidad de formular un diagnóstico coherente. En este sentido, hoy se dispone de un excelente material de auscultación que permite determinar con precisión, tanto

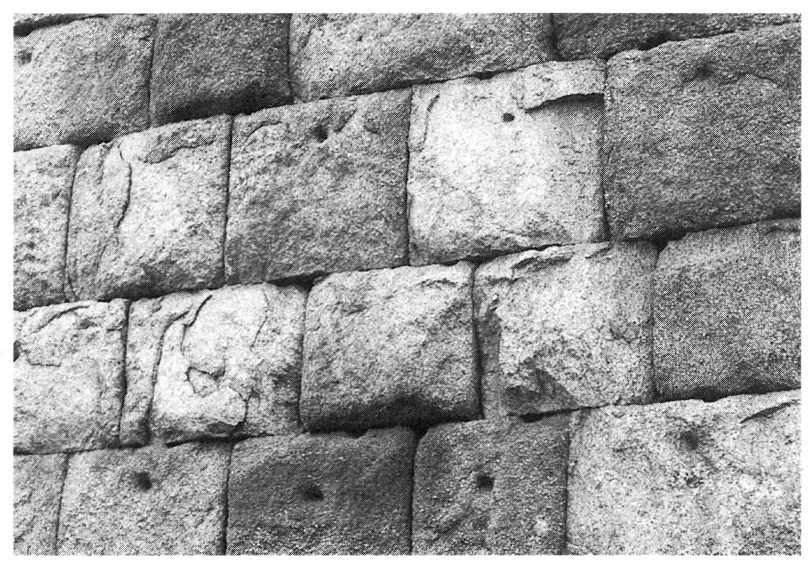

Fïg. 1.- I)etalle de la silleria del Acueducto de Segovia. (Fotografĩa del autor) su ancho y profundidad como su evolución (Fig. 2). Se cuenta con una información válida que nunca sustituirá un análisis de la obra por el proyectista.

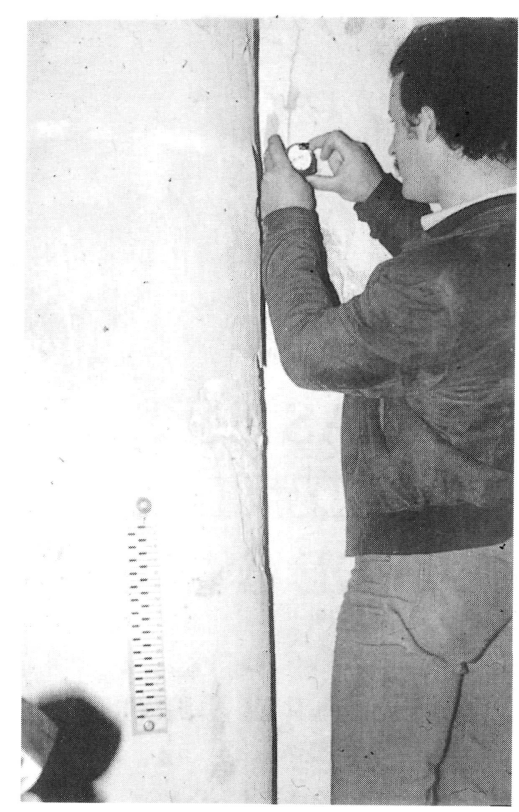

Fig. 2.- Auscultación de una fisura. San Hipólito de Támara. Palencia. (Fotografía del autor)

\section{SOBRE LAS FISURAS}

Cualquier actuación de reparación de una estructura pasa por un examen minucioso de su estado fisurativo. Gran parte de las estructuras que el arquitecto tieneque intervenir, en su quehacer rehabilitador, no precisan más que del entendimiento y la comprensión elemental del sistema de fuerzas. Así pues, hoy, nos encontramos con un arquitecto e ingeniero con formación en métodos analíticos, ya que se lo exige la propia herramienta que usa (el ordenador) y, sin embargo, parece haber dejado de lado la práctica de los métodos aludidos, basados en la geometría y la proporción, tan inherentes al arquitecto de todos los tiempos. No creemos que el cambio haya producido un resultado mejor (Fig.3, Bib.17).

Dependiendo de la forma en la que la estructura a rehabilitar se haya realizado tendremos estructuras de materiales cohesivos (Hormigón, acero, barro,..) o no cohesivos, estaremos en métodos estáticos (con enlaces suficientes para determinar las incógnitas) o hiperestáticos, la mayoría (con enlaces insuficientes). Una estructura de fábrica (adobe, ladrillo, piedra,...) es siempre hiperestática, ya que la solución del empuje de arco no está determinada y se mueve en un dominio. Este dominio, en un arco exento, en teoría, es el definido por el tercio central de la sección en toda su directriz que define el núcleo central, para que no existan tracciones entre dovelas. 


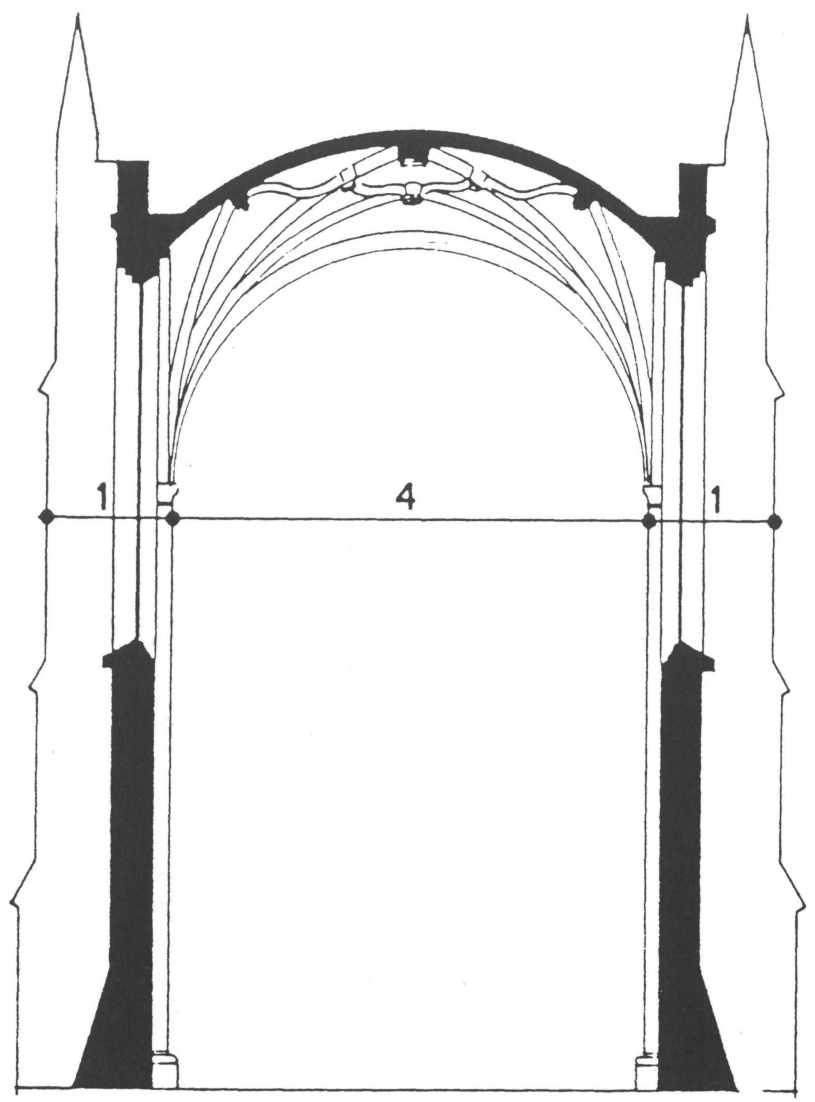

Fig. 3.- Proporción estable para una bóveda de crucería, según Rodrigo Gil de Hontañón.

Cualesquiera que sea el método de análisis a utilizar en la obra de rehabilitación, existirá una observación detenida, como parte del método. De esa observación, es difícil, por no decir imposible, sin instrumentación, saber cuál va a ser la deformación elástica o plástica de los elementos, pero sí podemos averiguarlo, a través de las fisuras donde los materiales han roto, o han manifestado distintos modos de trabajar.

Las fisuras indican roturas del material al asumir tracciones, oaparición de tracciones inadecuadas en puntos de la estructura para las que no están preparados, tales como las pastas de agarre en la obra de fábrica. La fisura, dicho de otro modo, es casi la única forma que tiene una estructura de expresar equilibrio y/o su nueva forma de ser estable.

Cuando no hubo herramientas de análisis, la estabilidad se garantizaba por correcciones al diseño de tipo empírico, basado, una vez, en medidas antropomórficas, otra, en trazados geométricos, todos ellos derivados, siempre, de la experiencia, pues, al lado del axioma o teorema está el consejo práctico.

Cualquier obra, pasa por el examen minucioso de su estado fisurativo. En 1748, Giovanni Poleni, en su "Memorie historiche della gran cupola del templo Vaticano", formalizó el primer estudio pericial en la conocida estructura de Miguel Ángel, por encargo del Papa Benedicto XIV, en un problema en donde, multitud de técnicos de prestigio, como Le Seur, Jacquier y Boscovich, no habían podido resolver cuál era, y hoy todavía es, el de la fisuración de la bóveda. Una bóveda de doble hoja hemisférica cargada fuertemente en clave. Poleni, resolvió el problema justificando que la bóveda era estable, pues contenía el funicular de su carga, en la hipótesis de rotura que manifestaban sus grietas. Todavía hoy, esta bóveda, sigue siendo motivo de análisis y experimentaciones en institutos y universidades (Fig.4. Bib.32).

Hay que tener en cuenta que una fisura, o grieta (fisura de grandes dimensiones), no es más perjudicial por ser mayor su amplitud (ancho a distancia medida según la normal entre labios), sino por su estado de evolución en el tiempo. Este hecho expresa que la respuesta de la estructura está cambiando a posiciones que pueden no ser estables.

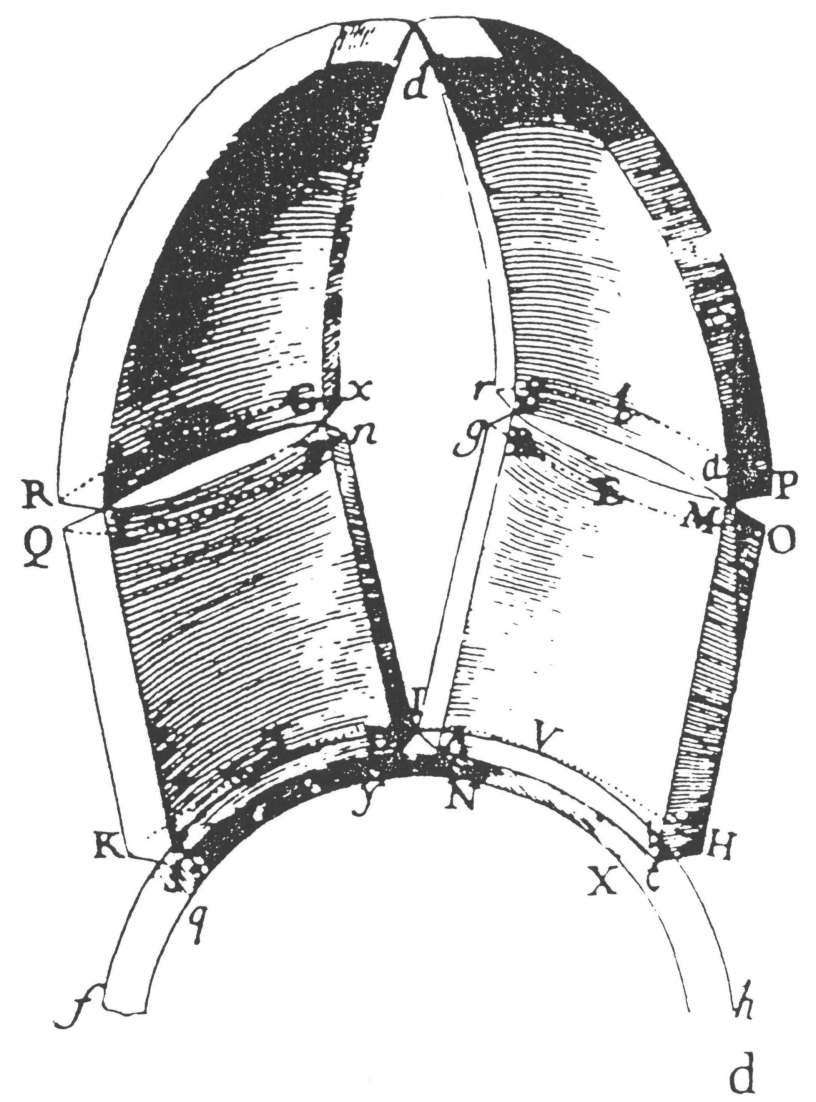

Fig. 4.- Hipótesis de colapso de la bóveda de San Pedro del Vaticano según G. Poleni.

\section{MOVIMIENTOS GLOBALES}

En las traslaciones no existe situación elástica especial, se trata de grietas totales, pero los estados fisurativos observados indican que existen partes del material que no trabajan y otras que lo hacen en demasía. Las grandes traslaciones de 
masas de un edificio indican cambios de posición, no sólo de las masas, cuanto de sus estados tensionales que producen incrementos de tensión que hay que verificar en cada caso. Estos estados pueden deberse a multitud de circunstancias y es preciso saber cuándo se está en condiciones de actuar. La intervención puede modificar los parámetros de rigidez del edificio (secciones, módulos de deformación, ...), creando situaciones de incompatibilidad y nuevos estados fisurativos no deseados. Es el caso, de la tendencia equivocada, de reforzar las estructuras de bóveda de piedra, hormigonando y armando su trasdós. En seguida aparecen estados de fisuración que hacen a la estructura más vulnerable. De aquí que sea tan importante conocer el objeto y su forma de funcionar para cuantificar el daño y aplicar su remedio (Fig.5).

Una traslación no debe confundirse con una deformación; ésta entraña, por definición, estados elásticos y/o plásticos que la primera no tiene. El cambio de posición topológica que implica una traslación puede ser consecuencia de infinitos estados de deformación elástica, pero de la traslación sólo interesan las nuevas posiciones de las masas en el equilibrio, no su historia anterior.

\section{APARICION DE LA FISURA}

La aparición de una fisura está condicionada al tiempo de aplicación de la carga y a la magnitud de aquélla. En toda estructura existe un determinado estado de tensión en cada punto. Cuando la distribución elástica es tal que, ninguna de las tensiones principales es nula, existe un llamado estado elástico triple. Las fisuras pueden ser obviadas desde un estado elástico doble de tensión o plano. El estado

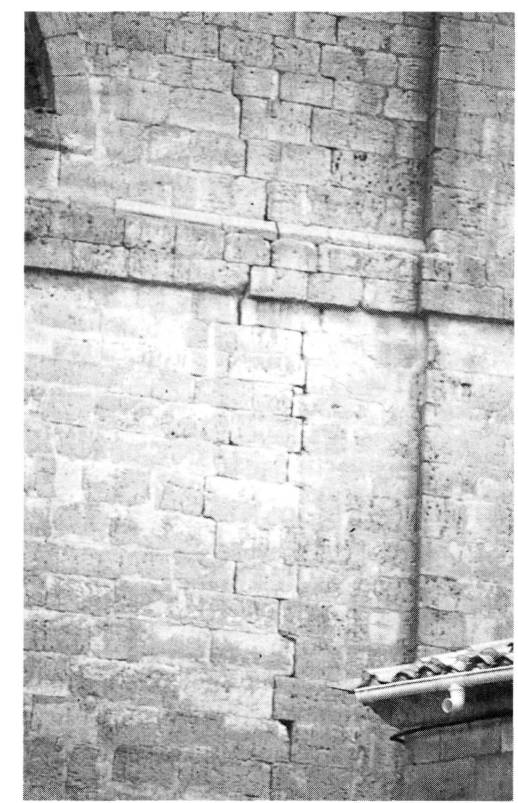

Fig. 5.- Movimientos de traslación global. San Hipólito de Támara. Palencia. (Fotografǐa del autor)

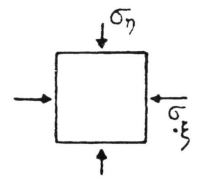

Eliptico.

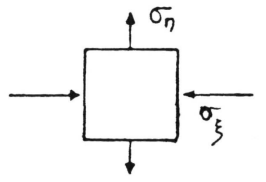

Hiperbólico.

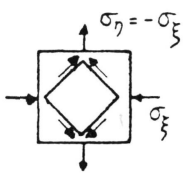

Cizallamiento puro.
Fig. 6.- Estados elásticos simples bidimensionales.

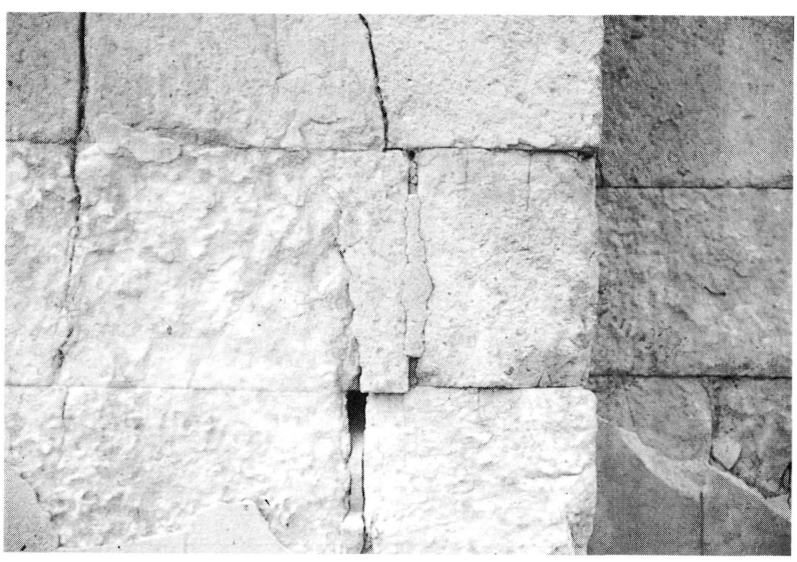

Fig. 7.- Fisura de agotamiento por cambio de fase en la piedra. Iglesia de Sta. Maria del Castillo,Fuentesauco Zamora. Arqto. A.Mas-Guindal. (Fotografía del autor)

plano contiene sólo dos tensiones principales, en las tres formas en las que puede manifestarse: elíptico, hiperbólico o cizallamiento puro (Fig.6).

\section{LA ALTERACIÓN DE LA FORMA DE TRABAJO}

Cualquier causa de fisuración refleja una discontinuidad del material y, por lo tanto, del sólido, causa que no siempre es debida a orígenes mecánicos, como condiciones de contorno, sino que puede ser debida a variaciones en las propiedades resistentes de los materiales, o cambios de fase (Fig.7). Variaciones de forma en un suelo pueden deberse a concentraciones de humedad, caso de las arcillas expansivas, donde su constitución laminar justifica los hinchamientos y cambios de volumen estacionales.

Los edificios antiguos están, básicamente, realizados con materiales cohesivos, es decir que no resisten tracciones, ello hace que la continuidad de sus geometrías esté confiada a su resultante de compresiones a través de los morteros y pastas de agarre, éstos son muy sensibles a cualquier tipo de movimiento. Los morteros, a lo largo de su vida en el edificio, han estado sujetos a muchos ciclos de humedad que les hacen pasar por dos estados. De una parte, el 
fraguado y, después, y mucho más lentamente, la carbonatación, a través de la cual el mortero pasa a hidróxido cálcico, reaccionando con el dióxido de carbono, formando carbonato cálcico. Esta patología es una de las grandes causas de inestabilidades globales, aun y cuando el material que unen, ladrillo y piedra, esté en buenas condiciones y la forma estructural haya sidobien concebida.

Un movimiento global entraña sólo una variación de posición, que puede estar ya estabilizada en el tiempo, por lo que la estructura habrá encontrado otra forma de equilibrio distinta de la original para la que fue pensada. La traslación expresa el resultado de un movimiento y debe ser observada en su estado final, comprobando que se ha llegado a una situación estable. Una fisura puede ser pequeña y manifestar, de forma elocuente, que existe un problema reciente en la estructura, simplemente por su capacidad de moverse y de evolucionar en el tiempo, creando situaciones potenciales de falta de estabilidad. Su observación y control merecen comentario aparte, pues constituyen toda una especialidad, pero en las imágenes presentamos sólo casos comunes.

De la forma que la estructura tenga una vez fisurada, va a desprenderse la idoneidad de aplicar un método de comprobación u otro. Un error tradicional, ha sido aplicar sistemáticamente métodos clásicos de análisis de estructuras de barras, de fundamentos elásticos, a estructuras de fábrica.

\section{LOS MÉTODOS}

Cualquier peritación o recálculo de una estructura fisurada requiere:

\section{-Una descripción formal y paramétrica del objeto a} estudiar.Luces, secciones, materiales, resistencias, ...etc.

-Un método para describir el campo de validez de las soluciones.

-Un método que describa una cualidad importante, que permita obtener un criterio de evaluación de la solución. (Descenso máximo, empuje máximo, carga de rotura,...etc.).

No puede arbitrarse un método único, ni siquiera recomendarse; sí nos permitimos, en cambio, hacer una pequeña distinción inicial, entre estructuras sin rigidez de constitución (estructuras de fábrica, abovedadas y murales) y estructuras con rigidez (estructuras de hormigón armado, acero laminado).

\subsection{El método geométrico}

Las estructuras de gravedad o de fábrica pueden peritarse por métodos basados en la geometría, ya que su estabilidad es una condición más restrictiva que su resistencia, por lo que los métodos vectoriales están aconsejados.

Estos métodos se basan en verificar, de forma geométrica y vectorial, el equilibrio estático de una estructura comprobando, de forma simple, su estabilidad global, por lo que son previos a actuaciones más especializadas.

Para ello, se utilizan los conceptos elementales del álgebra vectorial, asociando las acciones (cargas, sobrecargas y peso propio) de una estructura a vectores deslizantes, es decir, fuerzas con magnitud, dirección y sentido. Asi, la suma de varias fuerzas (vectores traslación), dará otra fuerza, que tiene como origen el de la primera y extremo el de la última. Esa suma de todas las fuerzas forma un polígono al que se le denomina polígono de fuerzas. Es condición para el equilibrio el que esté cerrado.

Teniendo en cuenta que existe una analogía geométrica entre las cargas, los esfuerzos cortantes y sus momentos flectores en relación a las curvaturas, los giros y las deformadas, se puede usar el polígono funicular para obtener las deformadas de piezasy, por lo tanto, las flechas.

El método gráfico tiene una utilidad para comprobar la estabilidad de estructuras de gravedad. Los arcos pétreos tienen esta condición y, en ellos, el conocimiento del valor y posición del empuje es fundamental para el diagnóstico.

En todo problema de peritaje de un arco pétreo, su estado de fisuración puede establecer conjeturas acerca de cuál es el modelo que está trabajando (Fig.8. Bib.22 y 23).
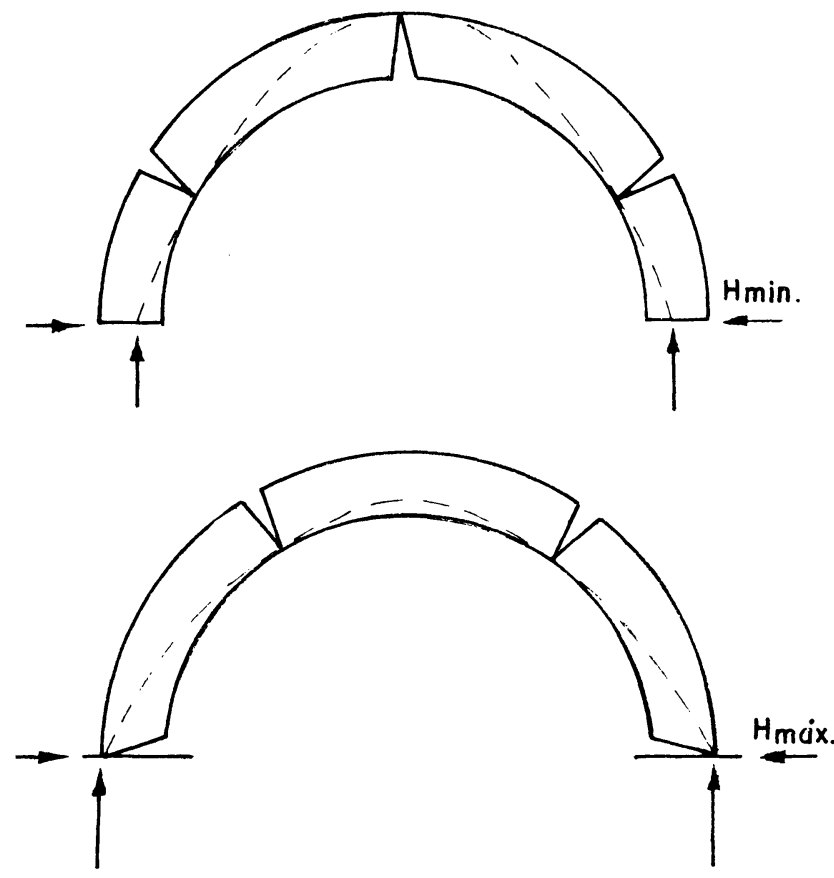

Fig. 8.- Modificación del empuje en un arco por descenso de la clave, según J. Heyman (Bib. 23). 
Teniendo en cuenta el efecto del descimbrado, es prácticamente imposible suponer que un arco de fábrica conserva su forma original transcurrido el tiempo (Fig.9).

Así pues, el arco teóricode fábrica tiene un nivel hiperestático alto, ya que existe una imposibilidad de poder definir con precisión cuál es el polígono de presiones y que son infinitos los que pasan por el núcleo central de inercia.

El proceso constructivo, o cualquier variación en las cargas, tienden a acomodar el sistema a una nueva forma de articulaciones definidas, como se verá.
El problema que se plantea en el proyecto es: dado un sistema de cargas, conocer cuál es la forma del arco que la resuelve y en el peritaje, dado una forma y material de un arco, verificar su estabilidad para un sistema de cargas.

El diseñador se encuentra con un primer problema: la estabilidad, trazar un polígono funicular (forma invertida del arco) que no se separe de la directriz del arco. Es fácil suponer que esto es imposible, ya que la directriz se corresponde con un sistema de cargas, si éstas varían, cosa normal en el uso de una estructura, dicha directriz lo hace variando de forma. Se trata entonces de encontrar una

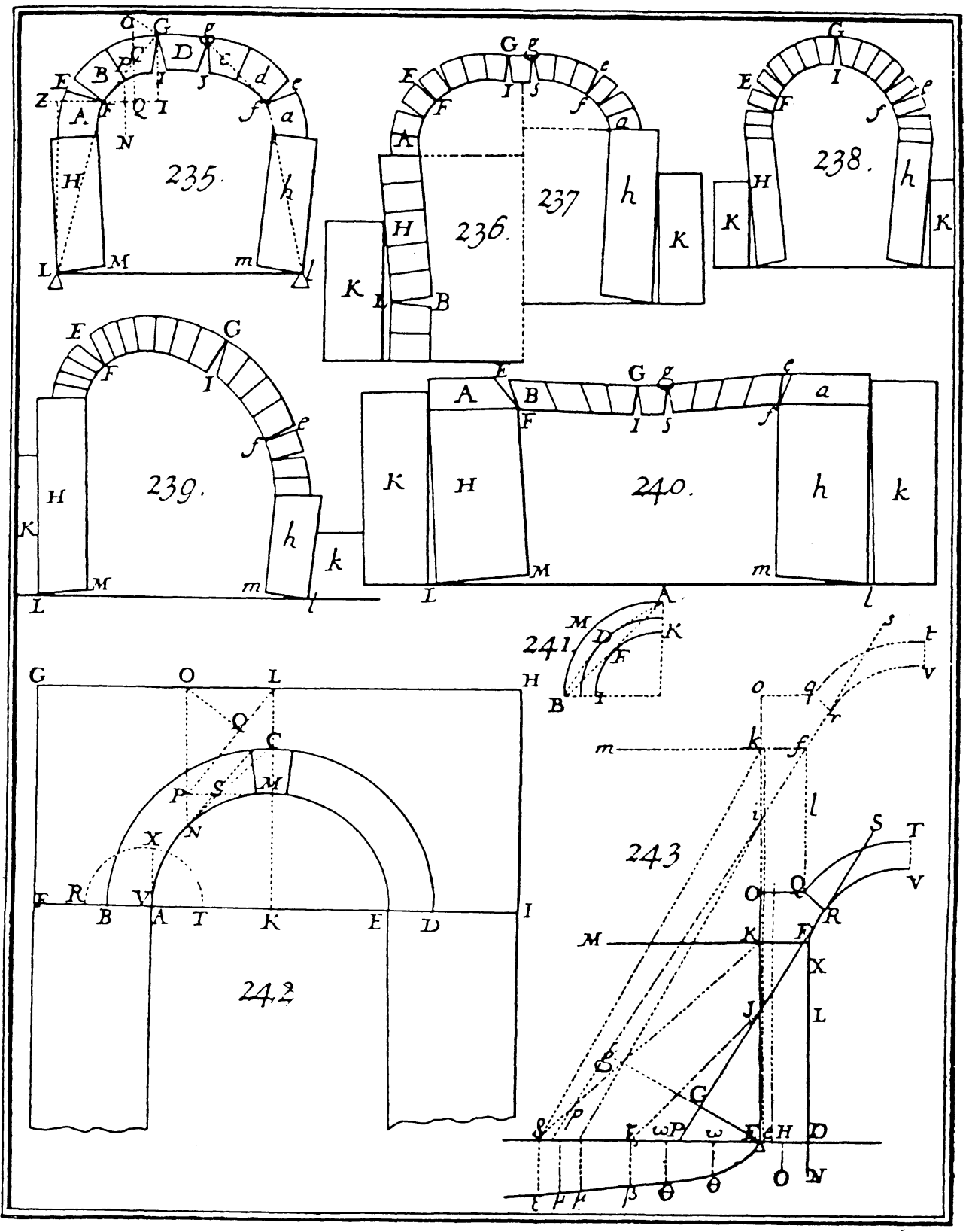

Fig. 9.- Tipos de rotura según Frezier (1737) (Bib. 16) 
directriz para el arco, cuyos núcleos centrales NC de sus secciones puedan asumir las variaciones de forma de la línea de empujes $L E$, ya que una fábrica no puede deformarse sin agrietarse (Fig. 10). Según se produzca la grieta en un arco, se posicionará la articulación, provocando mayor o menor empuje de respuesta.

\subsection{El método analítico}

Hasta hoy, numerosos problemas de resistenciade materiales han venido formulados en forma de ecuaciones. Cuando los problemas pueden ser resueltos por las ecuaciones de la

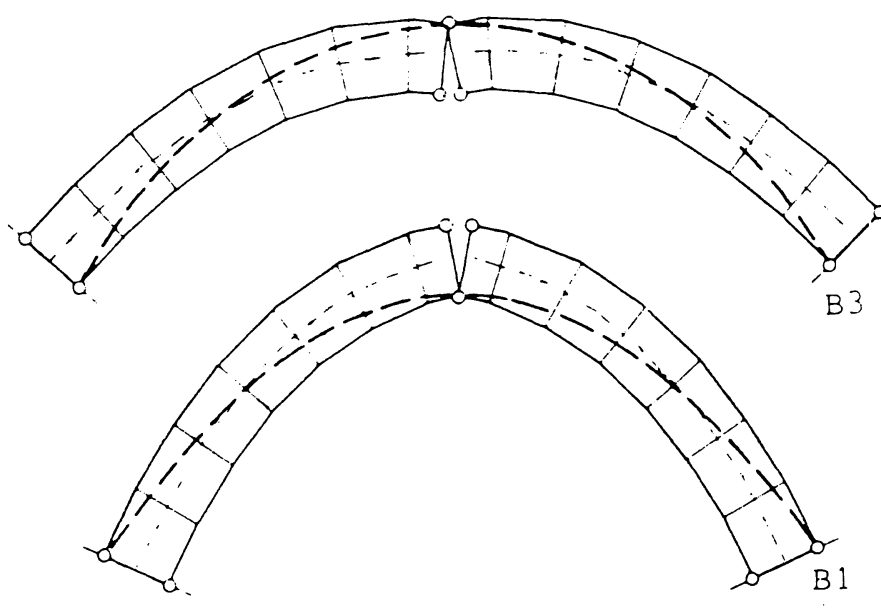

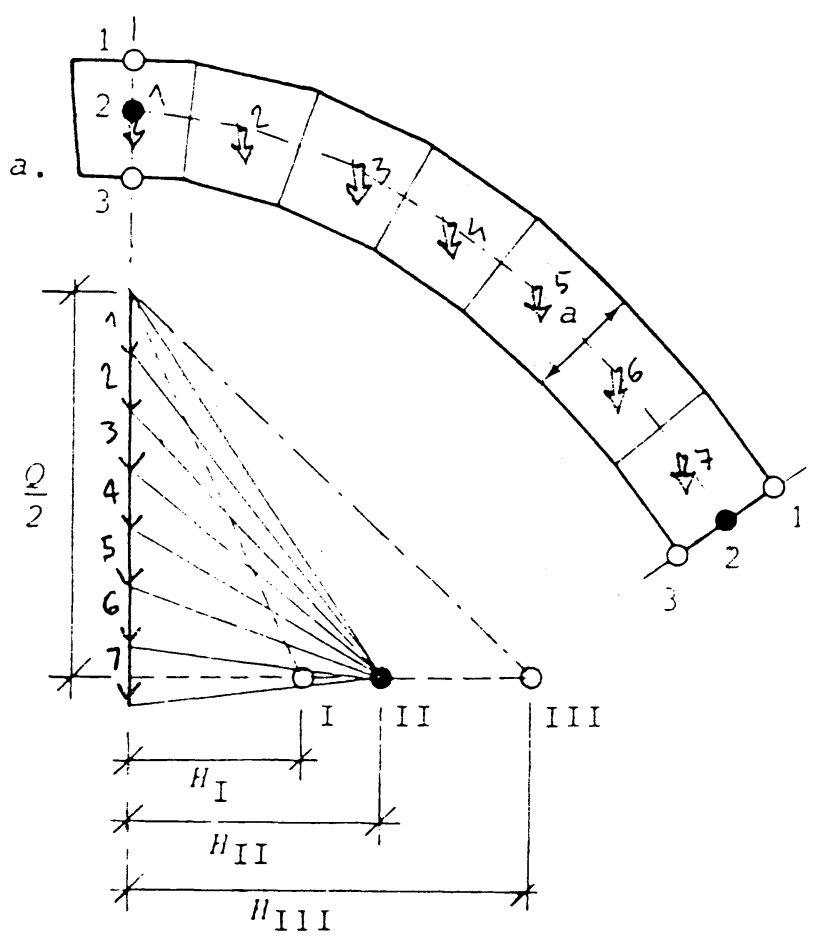

Fig. 10.- Lineas de presión de máximo y mínimo empuje compatibles con el sistema de cargas. estática, es el caso de la aplicación de las tres ecuaciones de equilibrio (casoanterior del método gráfico), el problema es de gran simplicidad. Gran parte de las operaciones de peritaje estriban en verificar el equilibrio del conjunto o de una parte.

Cuando las ecuaciones de equilibrio estático no son suficientes, hay que acudir a ecuaciones de compatibilidad entre tensiones y deformaciones. Este planteamiento tensional se formula para algunos casos en forma de ecuaciones diferenciales, cuya complejidad avanza con el problema. La solución de ecuaciones en derivadas parciales obliga a conocer la diferenciabilidad del problema, no siempre evidente y la determinación exhaustiva de las condiciones de contorno. Ecuaciones como las de la cuerda vibrante, la transmisión del calor, el calor, la catenaria, o la placa (ecuación de Lagrange), sólo admiten una familia reducida de soluciones, con frecuencia poco parecidas al caso que tenemos que resolver.

En resumen, este método trata, evidentemente, del cálculo diferencial. Caso de que la función que se maneja (trabajo, energía, deformación,..etc.) tenga diferenciabilidad suficiente, se puede calcular el óptimo de la función, por corresponder a un punto de derivada máxima, mínima o derivada segunda creciente. Un problema definido por una función es un problema controlado, en la medida que la función que lo define lo permita.

Siempre, la dificultad está, en que son pocos los problemas de estructuras fisuradas que se pueden definir de forma total desde una función.

\section{$6.3 \mathrm{EI}$ método de rotura}

Todo en realidad es arco, dada la imposibilidad de descomponer la fuerza que se quiere trasladar en dos normales a ella. Esto evidencia la naturaleza de la fuerza inclinada. Fuerza que coexiste aún en los sistemas adintelados, vigas, o sistemas tensados.

La cualidad fundamental reside en que, cargado verticalmente, produce compresiones oblicuas (empujes) en los apoyos, cuyas características, para arcos con rigidez a flexión, dependen de la clase de sustentación de que se disponga.

La piedra no soporta las tracciones, sin embargo, muy bien las compresiones. No obstante, si un montón de piedras no es estructura, sabiamente organizadas pueden ser estructura equilibrada, de forma que cada piedra transmite a la siguiente la carga que recibe.

Para apoyos simples, las reacciones y momentos dependen de la forma. Si los extremos impiden el movimiento, hay reacción horizontal. 
La posición de las cargas y su magnitud genera diferentes esfuerzos entre dovelas que si, como es normal en materiales no cohesivos (mampostería, sillería, etc.), no tienen resistencia a tracción, necesariamente cada arco, con una forma determinada, se diseña para unas cargas específicas que lo mantienen comprimido. Cuando esto no sucede, el arco se arruina. La línea de empujes o línea que une los puntos de aplicación de las cargas en las dovelas, suele acompañar la forma del arco. En dicho acompañamiento reside la sustancia del equilibrio.

Si una variación en las cargas ocasiona que la L.E. se salga del tercio central, aparecerán tracciones y, teóricamente, el arco no será estable. Pocas veces aparece el arco de piedra exento, ya que está cargado por muros en todo su trasdós, lo que hace que la línea de empujes pueda salirse de los estrictos límites del núcleo central de inercia y pueda tomar otras formas, haciendo participar a los elementos adyacentes al arco. Todo arco pétreo, en proceso de construcción, se realiza dovela a dovela, sobre cimbra. Al descimbrar, su clave, experimenta una pérdida de altura, por el acomodamiento de sus juntas, que suele provocar la fisuración de la parte inferior de la clave y exterior en apoyos. En realidad, esa sección girará como una rótula ocasionando un mecanismo que no sólo no produce el colapso, sino que puede favorecer el funcionamiento del arco. La existencia de fisuras en apoyos o clave sólo indica que el arco está resistiendo con una forma y modelo distinto del original, pero no por ello inválido. Si el empuje viene dado por $\mathrm{E}=$ Momento isostático/altura de la clave $\mathrm{h}$, al aumentar ligeramente $\mathrm{h}$, el empuje tiende a disminuir y el arco es más seguro (Fig. 10).

Estas rótulas pueden llegar a tres, cuatro formarian mecanismo, por lo que es un arte delimitar correctamente la posición de las rótulas en el peritaje.

Los arcos son estructuras que precisan la estabilidad de forma para su supervivencia. Cualquier modificación de forma (variación de forma o posición de apoyos), ocasiona líneas de empuje distintas que se salen de los pasos de carga definidos por los núcleos centrales.

El método plástico establece, a través de sus teoremas, que una línea adecuada de empujes que equilibre la carga y esté contenida dentro de la fábrica garantizará la estabilidad del arco bajo dicha carga. Lo que no queda claro es, qué estimación segura puede hacerse del arco, si las sobrecargas varían, como es habitual (viento, nieve, uso,...).

En los edificios de fábrica, el peso propio constituye el $80-90 \%$ de la carga total, hecho que va del lado de la seguridad de la estructura de fábrica, en la que el peso fijo ya es garantía de estabilidad, por lo que las fluctuaciones anteriores suelen ser permisibles dado el bajo nivel de trabajo. Por otra parte, aunque la aparición de una rótula implica un giro de dovelas (siempre que el giro no esté coaccionado) yuna concentración de tensión, en condiciones normales, el material puede soportar entre 20 y 30 veces más su tensión de trabajo sin romper, por lo que la formación de una rótula es posible y tolerable.

\subsection{El método numérico}

Los métodos numéricos permiten obtener soluciones particulares para problemas concretos. La solución se obtiene con suficiente aproximación, por lo que su aplicación está recomendada. Están basados en la aproximación a la solución a base de comprobaciones, dado que estas operaciones pueden ser realizadas por ordenadores con un coste relativamente bajo. Para su fiabilidad, exige una exhaustiva toma de datos, tanto en la geometría como en la definición del material (ecuación constitutiva) que se modeliza.

Entre estos métodos están, diferencias finitas, cálculo matricial y método de elementos fínitos. El primero de ellos, el de diferencias finitas, está basado en la realización de una aproximación finita de la derivada, resolviendo, así, problemas planteados en ecuaciones en derivadas parciales que no tienen solución, pudiendo, de esta forma, obtenerla, aunque sea de manera aproximada.

EI método matricial se basa en la forma que adoptan las relaciones que ligan los desplazamientos. Esta forma es lineal y permite un tratamiento matricial. Se basa en dos principios elementales, el de la linealidad y el de la superposición. De esta forma puede resolverse un problema tan sólo con determinar previamente cuál es el grado de definición del mismo y, por lo tanto, su nivel de aproximación.

Este método es indicado para la resolución de estructuras continuas, en donde es relativamente fácil suponer que no ha habido un cambio sustancial en el momento de inercia de las secciones. Su extensión, desde la energía, es el método de los elementos finitos, que hoy se presenta como la herramienta más poderosa que tiene la técnica del análisis estructural.

\subsubsection{Consideraciones sobre las bases de partida para el} análisis, por elementos finitos, en estructuras de fábrica

La obtención analítica de los estados de tensiones y deformaciones en estructurasfisuradas, que posteriormente van a ser reforzadas por inyección de mortero o barras, es de una gran complejidad, cuanto más, su reproducción analógica que permita la simulación de un fenómeno que depende de variables, cuya probabilidad de suceso es aleatoria, como son, por ejemplo, los derivados de la constitución del material base, como son la determinación de las discontinuidades de la roca base, o la elección de los planos de fractura entre otros o, en el caso del hormi- 
gón armado, la reconstrucción de la pieza elástica-plástica que está trabajando y la discursión latente entre giros plásticos, deformaciones elásticas y translaciones (Fig. 11).

En estructuras de piedra concurren una serie de problemas, no totalmente definidos, que pueden enumerarse en:

-No homogeneidad del material base por diversas causas. Como son distintas clases de rocas de constitución, que arrojan diversas patologías de pérdidas de resistencia y deformación.

- Variación de la posición de las líneas de fuerza (situación de las resultantes fuera de los núcleos centrales), como consecuencia de la evolución de la estructura a medida que aumenta la carga y comienza a aparecer la fisuración (fractura) (Bib.1).

-Comportamiento no lineal de las rocas y de sus zonas de interfase, debido a la plastificación de sección, problema éste difícil de cuantificar y del que existen muchos modelos, todavía hoy no todo lo deseables que los problemas requieren.

-En estructuras en donde no cabe más refuerzo que el de la inyección de barras,el comportamiento del acero laminado dentro de la estructura puede ocasionar concentraciones de tensión y discontinuidades en su distribución, en relación a las de la fábrica, hecho que se agrava en los casos en los que la piedra ha cambiado de fase por alteraciones químicas.

-En la piedra, las deformaciones de fluencia en las juntas son básicas, una vez producido el fenómeno de cambio de fase. La retracción es un problema subsidiario al anterior.

-Finalmente, problemas como la fractura súbita o deslizamiento de sillares, acompañado de la constante arenización de las pastas, puede colapsar una estructura sin aviso previo.

Finalmente, resulta estéril aplicar la técnica de análisis por elementos finitos a una estructura en la que previamente no se han realizado las hipótesis de trabajo. Dado que el material constitutivo de las fábricas no es continuo, al poner en carga la estructura, generalmente al descimbrar, aparecen tracciones, que deben adivinarse previamente, para predisponer el enlace adecuado. Aún así, la interpretación de los resultados no es tarea fácil y debe de permitir siempre reciclar sobre el modelo para obtener el nivel de afinamiento deseado en la solución.
La técnica por EF ha sido, desde 1960 hasta hoy, muy desarrollada a tenor de la cantidad y variedad de modelos que existen (Fig. 12).

Todos ellos están permitiendo desarrollar un conocimiento puntero de la forma de romper el material (mecánica de fractura) y la predicción de la rotura, que permite caracterizar, con más precisión cada vez, el camino que las fuerzas recorren dentro de la estructura, problema éste central del análisis estructural especializado.

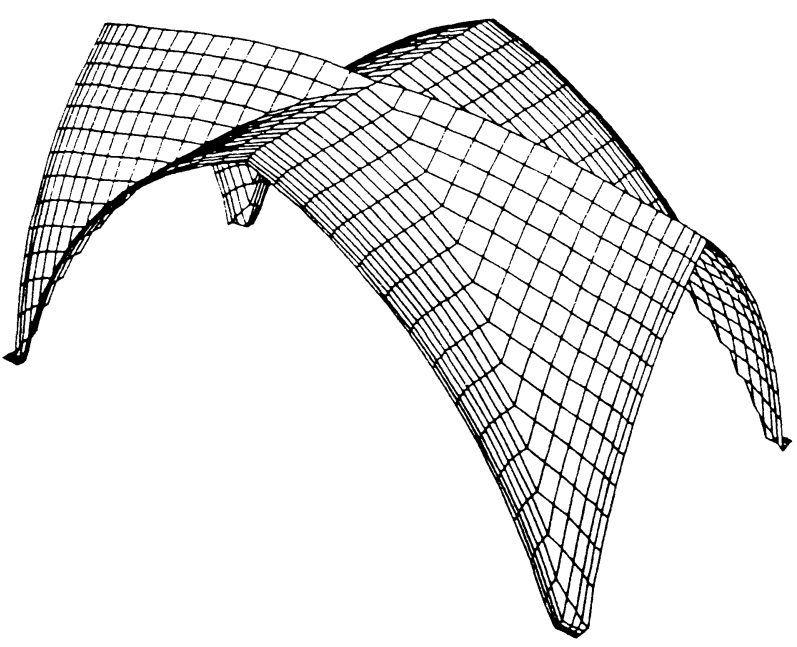

Fig. 11.- Modelo de Elementos Finitos de una bóveda de cruceria.

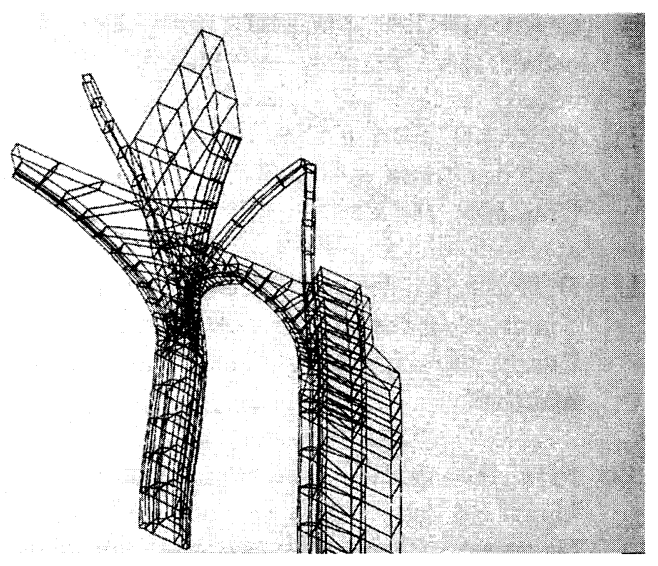

Fig. 12.- Deformación tridimensional magnificada por Elementos Finitos de la iglesia de San Sebastián de Villacastín (Segovia). Análisis de R. Corrés y el autor.

\section{BIBLIOGRAFÍA.}

(1) ABRAHAN, POL: "Les Données plastiques et fonctionelles du problème de l'ogive".- Recherche $n^{\circ} 1$ : Le Problème de l'ogive, Paris, Centre International des Instituts de Recherche, 1939, pp 29-51, 7 figs. * "Violet-le-Duc et le rationalisme médiéval", Paris, Vincent
Fréal et Cie, 1934. 116 pp., 49 figs.

* "Viollet-le-Duc et le rationalisme médiéval", Bulletin 
monumental, Paris, A. Picard, 1934. vol XCIII, pp. 69-88, 1 illus.

(2) ADHÉMAR, JOSEPH: "Traité de la coupe des pierres", Paris, V. Dalmont, 5 a édition, 1856

* "Traité de charpente", Paris, Carilian Goeury et V. Dalmont, $2^{\mathrm{e}}$ édition, 1854.1 vol. text; atlas, 48 plates.

(3) ANDREWS, FRANCIS B: "The Medieval Builder and His Methods, Oxford, University Press, 1925, 99 pp., 3 fig., 13 plates.

(4) ANFRAY, MARCEL: "Les Architectes des cathédrales", Les Cahiers téchniques de l'art, tome I, fasc. 2, mai-déc 1947, pp. 5-16.

(5) ATWOOD, GEORGE: "A Dissertation on the Construction and Properties of Arches", London, Lunn: Egerton, 1801.

(6) BLAKE, MARION ELIZABETH: "Ancient Roman Construction in Italy from the Prehistoric Period to Augustus", Washington D.C., Carnegie Institute of Washington, Publication $\mathrm{n}^{\circ}$ 570, 1947.

(7) BLANCHARD, EDMÉ: "Traité de la coupe des bois pour le revêtement des voûtes, arrières-voûtes, trompes, rampes et tours rondes ...", Paris, J. Josse, 1729. 46 plates.

(8) BRANNER, ROBERT: "Three Problems from Villard de Honnecourt Manuscript", The Art Bulletin, Mar. 1957, vol. XXXIX, pp. 61-66.

(9) CHOISY, AUGUSTE: "L'Art de bâtir chez les Byzantins", Paris, Librairie de la Société Annonyme de Publications Périodiques, 1883

(10) COLOMBIER, PIERRE DU: "Les Chantiers des cathédrales", Paris, A. et J. Picard, 1953.

(11) DERAND, FRANÇOIS: "L'Architecture des voûtes ou l'art des traits et coupe des voûtes", Paris, Sebastien Cramoisy, 1643.

(12) DUPUIT, J: "Traité de l'équilibre des voûtes et de la construction des ponts en maçonnerie", Paris, Imprimerie Cusset et Cie; Librairie Dunod, 1873.398 pp. et atlas de 49 planches.

(13) FITCHEN, JOHN: "A Comment on the Function of the Upper Flying Buttress in French Ghotic Architecture", Gazette des Beaux-Arts, Feb. 1955, ser. 6, vol XIV, pp. 6990 .

(14) FLETCHER, SIR BANISTER: "A History of Architecture on the Comparative Method", New York, Scribner's many edns, various dates.

(15) FRANKLIN, ALFRED: "Dictionnaire historique des arts, métiers et professions exercés dans Paris depuis le XIII siècle, Paris, H. Welter, 1906.

(16) FREZIER, AMÉDÉE FRANÇOIS: "La Théorie et la pratique de la coupe des pierres et des bois, pour la construction des voûtes et autres parties des bâtiments civils et militaires, ou traité de stéréotomie de l'architecture", 3 vols., Strasbourg, J.D. Doulsseker le fils, 1737-9.

(17) GARCIA, SIMÓN: "Compendio de arquitectura y simetría de templos". Madrid Biblioteca Nal. de Madrid Cap.1-7, 1544-1554.

(18) GAUTHIER, J.L: "Cours de matériaux et éléments de construction: Croquis de stéréotomie" (École Nationale Supérieur des Beaux-Arts), Paris, Éditions Vincent Fréal et $\mathrm{Cie}$, s.d. 60 plates.

(19) GAUTIER, HUBERT: "Traité des ponts et chaussées ...", Paris, André Cailleau, 1716, 131 pp., 28 plates. Vide Particularly: chap. XIV, pp. 68-71, "Des Échafaudages"; chap. XV, pp. 71-77, "Des Cintres, Mortoises, \& Poutres armées"; and the nine examples of centering frames on plate XVIII.

(20) GUSTAVINO, RAFAEL: "Essay on the Theory and History of Cohesive Construction Applied Especially to the Timbrel Vault”, Boston, Ticknor \& Co., 1892. 149 pp. 62 figs.

*"Prolegomenos on the Function of Masonry in Modern Architectural Structures", part I (New York, Record \& Guide Press, Printers, 1896).

(21) GRUNAU, EDUARD B.: "Lesiones en los edificios". Barcelona, CEAC, 1988

(22) HEYMAN, J. HORNE. \& BAKER: "The stone skeleton", Cambridge, U. Press, 1956.

(23) HEYMAN, J: "Estructuras de fábrica". Madrid, Inst. Juan de Herrera, 1995.

(24) KUBLER, GEORGE: "A Late Ghotic Computation of Rib Vault Thrusts", Gazette des Beaux.Arts, 1944, series 6 , vol. Xxvi, pp. 135-48, 6 illus.

(25) JOISEL, ALBERT: "Fisuras y grietas en morteros y hormigones”. Barcelona, Ed. Técnicos Asoc., 1969.

(26) MAS-GUINDAL LAFARGA, ANTONIO-JOSÉ: "Aproximación al análisis de piezas rectas de hormigón armado en estado último", Tesis Doctoral 1981 .E.T.S.A.Madrid. 2. Tomos.

(27) MAS-GUINDAL LAFARGA, ANTONIO-JOSÉ: "Master en restauración de monumentos. La estructura en la historia de la arquitectura". Madrid, Universidad de Alcalá de Henares, 1996.

(28) MASTRODICASA, SIXTO: "Dissesti statici delle structure edilizie”, Milán, Hoepli, 1974.

(29) OREN VILNAY \& SUN-SUM CHEUNG: "Stability of masonry arches", ASCE Journal of Structure Eng. $n^{\circ} 10 / 86$

(30) OTTOHENKEL: “Estática Gráfica”,Barcelona, Ė̃. Labor, 1956 
(31) PLANAT, PAUL AMÉDÉE: “Encyclopédie de l'architecture et de la construction" (Bibliothèque de la Construction Moderne), 6 vols., Paris, Dujardin et Cie. Éditeurs, 1888-93, Vide Particulary the following articles:

* "Levage", vol. V, pp. 369-98 (incl. figs. 46-59 on rolling scaffoldings).

* "Matériel de Cantier, III: Échafauds", vol. v, pp. 619-33, 31 figs.

(32) POLENI, G.: "Memorie historiche della gran cupola del Templo Vaticano". (Padua: Nella Stamplia del Seminario 1748. Facsímil castellano INTEMAC, Madrid, 1983.

(33) RUSSO, CRISTOBAL: "Lesiones en los edificios", Barcelona, Salvat Ed., 1934.

(34) RONDELET, JEAN: “Traité théorique et pratique de l'art de bâtir", Paris, L'auteur, 1812-17. 6 vols, text, 2 vols. (of 192 folding) plates.

(35) SALVADORI, MARIO G.: "Thin Shells", Architectural Record, New York, F.W. Dodge, July 1954, vol. CXVI, pp. 173-9, 28 figs.
(36) SÉJOURNÉ, PAUL: "Grandes voûtes", 6 tomes in 3 vols., Bourges, Imprimerie V ve Tardy-Pigelet et Fils, 1913-16.

(37) SIMSON, OTTO VON: "The Gothic Cathedral", New York, Bollingen Foundation (Bollingen Series XLVIII), 1956.

(38) VIOLLET-LE-DUC, EUGÈNE EMMANUEL: "Dictionnaire raisonné de l'architecture française du XI" au $X V I^{e}$ siècle, Paris, Librairies-Imprimeries Réunies. 1858-68, 10 vols. Vide Particulary the following articles:

\footnotetext{
"Armature", vol. I, pp. 461-6

"Chaînage", vol. II, pp. 396-404

"Charpente", vol. III, pp. 1-58

"Construction", vol. IV, pp. 1-279

"Échafaud", vol. V, pp. 103-14

"Engin", vol. V, pp. 210-69

"Escalier", vol. V, pp. 287-331

"Étai", vol. V, pp. 332-45

"Galerie", vol. VI, pp. 8-21

"Voûte", vol. IX, pp. 464-550
}

(*) "De la construction des monuments religieuses en France" Annales archéologiques, Paris, Librairie Didron, vol. VI, avr. 1847, chap. v, pp. 194-205, 17 illus. 